\title{
Toll-like receptor 4 on islet $\beta$ cells senses expression changes in high-mobility group box 1 and contributes to the initiation of type 1 diabetes
}

\author{
Min $\mathrm{Li}^{*}$, Lujun Song*, Xiaodong Gao, \\ Wenju Chang and Xinyu Qin ${ }^{1}$ \\ Department of General Surgery \\ Zhongshan Hospital \\ Shanghai Medical School \\ Fudan University \\ Shanghai 200032, China \\ ${ }^{1}$ Corresponding author: Tel, 86-21-64037224; \\ Fax, 86-21-64037224; E-mail, qin.xinyu@ zs-hospital.sh.cn \\ *These authors contributed equally to this work. \\ http://dx.doi.org/10.3858/emm.2012.44.4.021
}

Accepted 2 January 2012

Available Online 5 January 2012

Abbreviations: DTZ, dithizone; ECL, enhanced chemiluminescence; HMGB1, high-mobility group box 1; NHS, N-hydroxysuccinimide; NOD, non-obese diabetic; PAMPs, pathogen-associated molecular patterns; RAGE, advanced glycation end products; SLE, systemic lupus erythematosus; T1DM, type 1 diabetes mellitus; TLR, toll-like receptor

\begin{abstract}
Type 1 diabetes mellitus is caused by the autoimmune destruction of $\beta$ cells within the islets. In recent years, innate immunity has been proposed to play a key role in this process. High-mobility group box 1 (HMGB1), an inflammatory trigger in a number of autoimmune diseases, activates proinflammatory responses following its release from necrotic cells. Our aim was to determine the significance of HMGB1 in the natural history of diabetes in non-obese diabetic (NOD) mice. We observed that the rate of HMGB1 expression in the cytoplasm of islets was much greater in diabetic mice compared with non-diabetic mice. The majority of cells positively stained for toll-like receptor 4 (TLR4) were $\beta$ cells; few $\alpha$ cells were stained for TLR4. Thus, we examined the effects of anti-TLR4 antibodies on HMGB1 cell surface binding, which confirmed that HMGB1 interacts with TLR4 in isolated islets. Expression changes in HMGB1 and TLR4 were detected throughout the
\end{abstract}

course of diabetes. Our findings indicate that TLR4 is the main receptor on $\beta$ cells and that HMGB1 may signal via TLR4 to selectively damage $\beta$ cells rather than $\alpha$ cells during the development of type 1 diabetes mellitus.

Keywords: diabetes mellitus, type 1; HMGB1 protein; islets of Langerhans; mice, inbred NOD; toll-like receptor 4

\section{Introduction}

Type 1 diabetes mellitus (T1DM), also known as insulin-dependent diabetes mellitus or juvenile diabetes, is generally believed to be caused by an interaction between the immune system and an intricate network of environmental and genetic factors (Pino et al., 2010). The key feature of T1DM is cell-mediated destruction of insulin-secreting $\beta$ cells in the pancreatic islets (Giarratana et al., 2004).

HMGB1 was initially identified nearly $30 \mathrm{yr}$ ago as a nuclear protein that is important for transcriptional regulation. HMGB1 causes DNA bending and facilitates the binding of several regulatory protein complexes to DNA (Zhang et al., 1999; Lotze and Tracey, 2005; Li et al., 2010). In addition to its role in transcriptional regulation, HMGB1 has been shown to activate proinflammatory responses following its release by necrotic cells into the extracellular environment (Wang et al., 2010). HMGB1 is also implicated in the pathogenesis of a number of diseases associated with inflammation and tissue injury (Wang et al., 2010). Recently, many studies have suggested that HMGB1 acts as an inflammatory trigger in autoimmune diseases such as rheumatoid arthritis and systemic lupus erythematosus (SLE) (Wittemann et al., 1990; Abdulahad et al., 2010).

Several important receptors have been implicated in HMGB1 signaling, including members of the toll-like family of receptors (TLRs) and the receptor for advanced glycation end products (RAGE) (Klune et al., 2008). The TLR family is an evolutionarily conserved component of the innate immune system 
(Medzhitov, 2007). The recent discovery of TLRs and studies performed in animal models of the disease led to the hypothesis that the innate immune system plays a key role in triggering the autoimmune process (Meyers et al., 2010). RAGE, which specifically binds advanced glycation end products, is a member of the immunoglobulin superfamily of cell surface receptors (Song et al., 2011). HMGB1 may signal TLRs and RAGE to activate nuclear factor- $\kappa B$ (NF- $\kappa B$ ), which induces the production of proinflammatory cytokines and the upregulation of leukocyte adhesion molecules, thereby promoting injury and inflammation (Van Beijnum et al., 2008; Nogueira-Machado et al., 2011).

It is well known that $\alpha$ cells are not affected during the development of diabetes, whereas $\beta$ cells undergo extensive destruction, even though $\alpha$ cells form a ring and provide a physical barrier around the $\beta$ cells. In the present study, the distribution of HMGB1 in the pancreas was examined by immunohistochemical staining, which demonstrated that the the rate of HMGB1 expression in the cytoplasm of islets was much greater in diabetic mice compared with non-diabetic mice. The non-obese diabetic (NOD) mouse develops a spontaneous form of autoimmune diabetes that mimics many features of the human disease and typically develops at 12 to 14 weeks of age (Anderson and Bluestone, 2005). We tested our hypothesis that distinct HMGB1 receptors are distributed on different pancreatic cell types in NOD mice. Thus, we examined the effects of anti-TLR2, anti-TLR4, anti-TLR9 and anti-RAGE antibodies on HMGB1 cell surface binding, which confirmed that HMGB1 interacts with TLR4 in isolated islets. These changes in HMGB1 and TLR4 were detected throughout the course of diabetes, being significantly upregulated in the early stages of diabetes and then slowly decreasing over time. Our data provide strong evidence that TLR4 is the main receptor on $\beta$ cells and that HMGB1, which is passively released from damaged islet cells, acts through the TLR4 signaling pathway to selectively damage $\beta$ cells rather than $\alpha$ cells during the development of autoimmune diabetes.

\section{Results}

\section{HMGB1 is associated with islet destruction}

Because it has been reported that HMGB1 can be passively released from damaged cells (Abdulahad et al., 2010) or necrotized tissues (Lin et al., 2011a, $2011 \mathrm{~b}$ ), we examined whether it is also released from damaged islet cells in T1DM using NOD mice. Histological and morphological assessment of
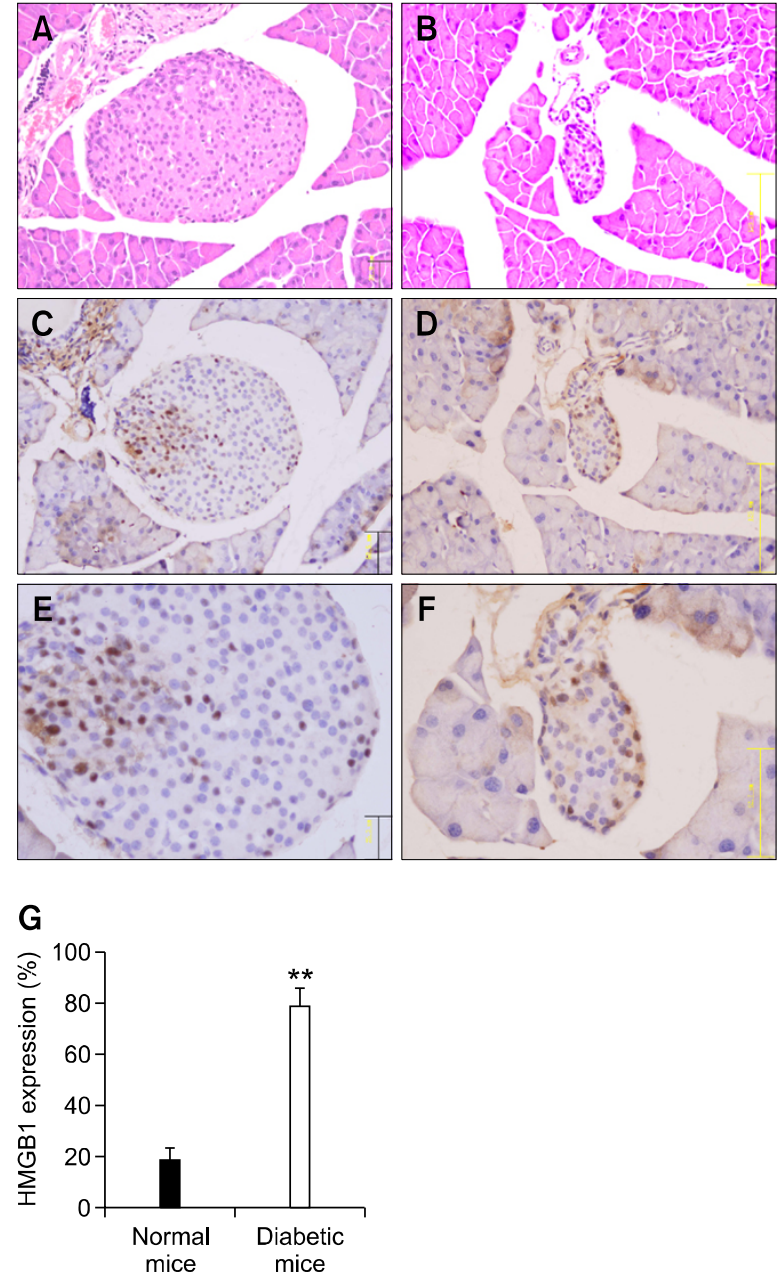

Figure 1. Hematoxylin and eosin staining of pancreatic sections demonstrates extensive islet destruction in diabetic NOD mice (B) compared with 4-week-old, non-diabetic NOD mice (A). Immunohistochemical staining shows preferential localization of HMGB1 in the nuclei of islet cells in 4-week-old non-diabetic mice (C, E). HMGB1 expression in the cytoplasm of the islets is much higher with the development of diabetes $(D, F)$. The rate of HMGB1 expression in the cytoplasm of islets was much greater in diabetic mice compared with non-diabetic mice $(\mathrm{E}){ }^{*} P<0.01(n=9$ per group).

pancreatic sections stained with hematoxylin and eosin indicated extensive islet destruction (Figure 1B) in diabetic NOD mice compared with 4-week-old non-diabetic mice (Figure $1 \mathrm{~A}$ ). We also examined the expression and cellular distribution of HMGB1 in the specimens of NOD pancreatic tissues by immunohistochemical staining. HMGB1 was mainly localized in the nuclei of islet cells in 4-week-old non-diabetic NOD mice (Figure 1C). However, HMGB1 increased in the cytoplasm of islets with the progression of diabetes (Figure 1D). The rate of HMGB1 expression in the cytoplasm of islets was much greater in diabetic mice compared with nondiabetic mice $(P<0.01)$ (Figure 1E). Taken together, 

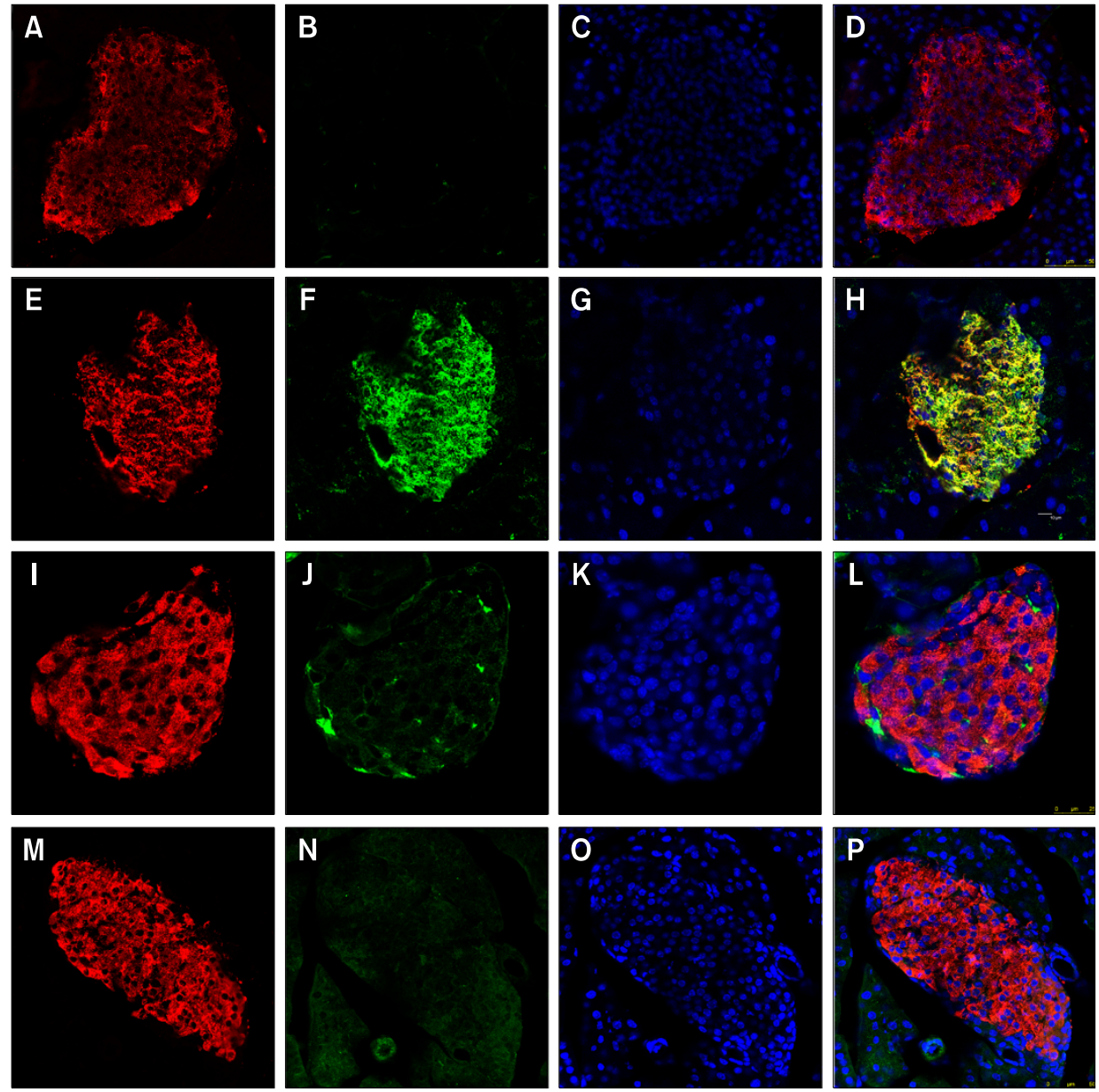

Figure 2. Expression of HMGB1 receptors (TLR2, TLR4, TLR9 and RAGE) and insulin in pancreatic islets of 4-week-old non-diabetic NOD mice. (A, E, I, M) Insulin immunostaining (red) of $\beta$ cells. (B, $F$, J, N) TLR2, TLR4, TLR9 and RAGE immunostaining (green). (C, G, K, O) DAPI nuclear staining (blue). Pancreatic islets from 4-week-old non-diabetic NOD mice show little or no expression of TLR2 (B), TLR9 (J) or RAGE (N). In contrast, TLR4 is highly expressed on the islets (green) in 4-week-old non-diabetic NOD mice (F).Co-localization (yellow) of TLR4 and insulin $(\mathrm{H})$ suggested that TLR4 is mainly expressed in $\beta$ cells. our data suggest that HMGB1 may be passively released from damaged islet cells or inflamed islet cells during autoimmunity.

\section{Expression of HMGB1 receptors on the pancreatic islets of NOD mice}

The expression and cellular distributions of HMGB1 receptors, including TLR2, TLR4, TLR9 and RAGE, in the pancreatic islets of NOD mice were examined by immunofluorescence and visualized by confocal microscopy. Little or no expression of TLR2, TLR9 or RAGE was observed in the pancreatic islets of 4-week-old, non-diabetic NOD mice (Figures 2B and $2 \mathrm{~J}$ and $2 \mathrm{~N}$ ). In contrast, TLR4 was mainly localized in the islets and indicated increased expression in 4-week-old non-diabetic NOD mice (Figure 2F).

Next, we investigated which of the pancreatic cell types were positive for TLR4 receptors. We performed double-labeling for islet $\alpha$ cells and $\beta$ cells separately with TLR4 in 4-week-old non-diabetic NOD mice. TLR4 was mainly distributed in the cytoplasm.
Furthermore, the cells expressing TLR4 were insulin-positive cells (i.e., $\beta$ cells), which comprise the majority of cells in the islet (Figures 2E-2H). The glucagon-positive cells ( $\alpha$ cells) formed a ring around the islet; however, relatively few $\alpha$ cells expressed TLR4 (Figure 3).

\section{HMGB1 interacts with TLR4 in isolated islet cells}

To further study the interactions between HMGB1 and its corresponding receptors, we examined the effects of anti-TLR2, anti-TLR4, anti-TLR9 and anti-RAGE antibodies on HMGB1 cell surface binding in islets using confocal microscopy. Islets were isolated from 4-week-old non-diabetic NOD mice and purified by handpicking. The dispersed islet cells were then cultured in a standard medium. Cell surface binding of $\mathrm{N}$-Hydroxysuccinimide (NHS)-fluorescein-HMGB1 was observed in islet cells incubated with NHS-fluorescein-HMGB1 for 6 $\mathrm{h}$ at $4^{\circ} \mathrm{C}$, and the staining formed an annular pattern (Figure 4A). Pretreatment with anti-TLR2, anti-TLR9, anti-RAGE or $\lg$ did not significantly influence 

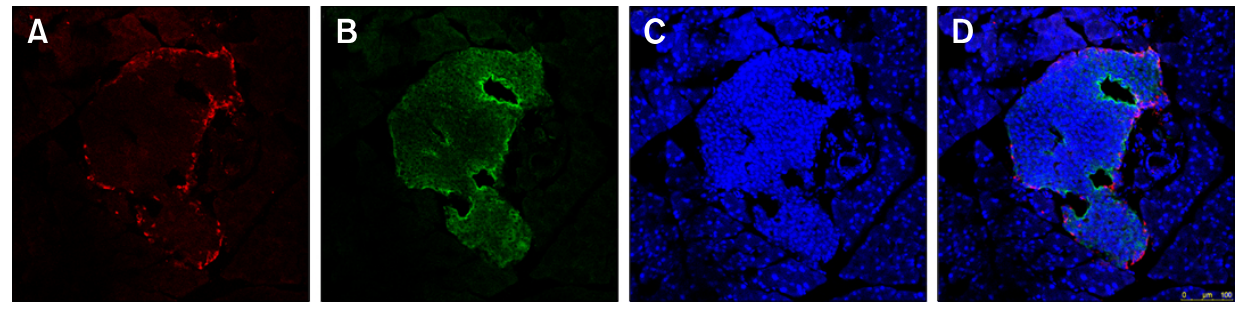

Figure 3. TLR4 is not expressed in $\alpha$ cells. Islets from 4-week-old non-diabetic NOD mice were double-labeled with TLR4 and glucagon. (A) Glucagon immunostaining (red). (B) TLR4 receptor immunostaining (green). (C) DAPI nuclear staining (blue). (D) Co-localization of TLR4 and glucagon indicate sparse expression of TLR4 in $\alpha$ cells.
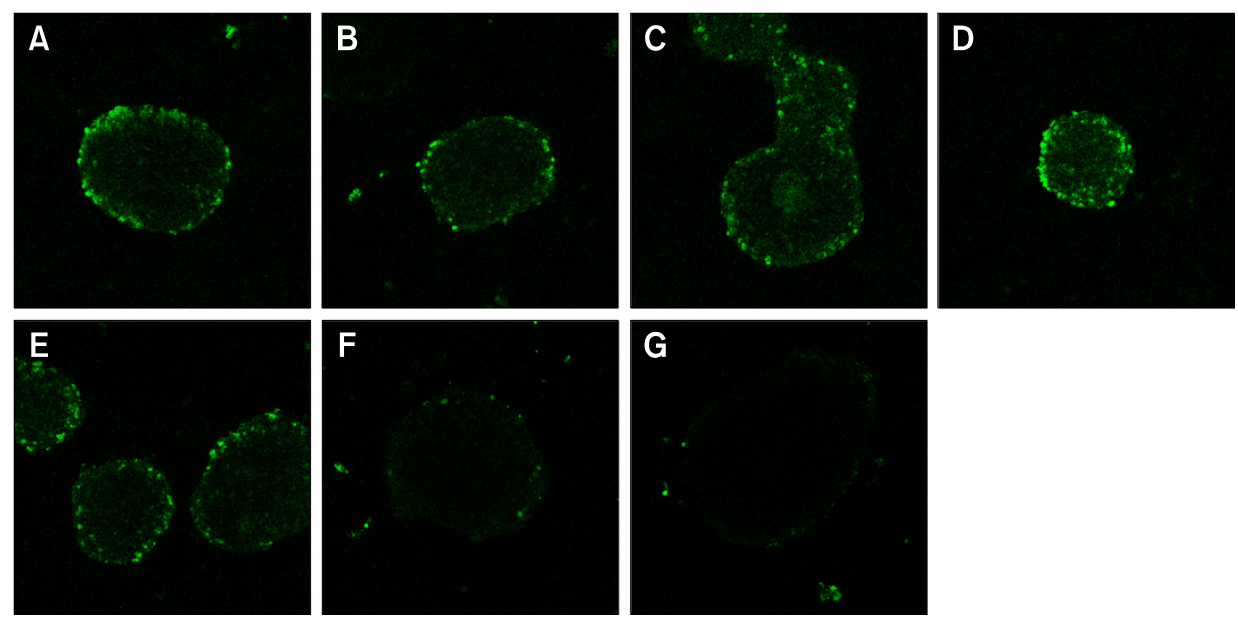

Figure 4. Effects of TLR antibodies on cell surface binding of HMGB1. Islets were isolated from 4-week-old non-diabetic NOD mice, plated in six-well plates and used at 70\% confluence. (A) Incubation of islets with NHS-fluorescein-HMGB1 for $6 \mathrm{~h}$ at $4^{\circ} \mathrm{C}$ resulted in an annular staining pattern. (B-G) Cells were also preincubated with $25 \mu \mathrm{g} / \mathrm{mL}$ of anti-TLR2 (B), TLR9 (C), RAGE (D), IgG (E), TLR4 (F) or unlabeled HMGB1 (G) for 30 min at $4^{\circ} \mathrm{C}$ in Opti-MEM I medium, followed by $5 \mu \mathrm{g} / \mathrm{mL}$ NHS-fluorescein-labeled HMGB1 for $6 \mathrm{~h}$ at $4^{\circ} \mathrm{C}$. Cells were then washed three times with PBS (pH 7.2) and fixed for $20 \mathrm{~min}$ at room temperature in $2 \%$ paraformaldehyde-PBS. After fixing, fluorescence was recorded with a confocal microscope.

HMGB1 cell surface binding (Figures 4B-4E). However, anti-TLR4 antibodies (Figure 4F) or unlabeled HMGB1 (Figure 4G) decreased HMGB1 cell surface binding, visualized by a reduction in cell-associated fluorescence intensity compared with IgG-treated controls. These results indicate that HMGB1 physically interacts with TLR4 in islet cells.

\section{HMGB1 and TLR4 protein expression in the pancreas of NOD mice}

Pancreatic HMGB1 and TLR4 protein expression was evaluated by western blotting at various times in the natural history of diabetes in NOD mice (Figure 5). Pancreatic expression of both HMGB1 and TLR4 was low in young NOD mice (4-6 weeks of age). In contrast, the pancreatic expression of HMGB1 and TLR4 was significantly upregulated in the early stage of diabetes (10-14 weeks of age) $(P$ $<0.01)$. To our surprise, in older diabetic mice (28-32 weeks of age), the protein expression of
HMGB1 and TLR4 was markedly downregulated ( $P$ $<0.01)$.

\section{Changes in pancreatic HMGB1 and TLR4 mRNA expression during the development of diabetes in NOD mice}

We assessed pancreatic HMGB1 and TLR4 mRNA expression every 3-5 weeks during the development of diabetes in NOD mice (Figure 6). This analysis showed no marked changes in HMGB1 mRNA expression in the first 8 weeks of life of NOD mice. However, HMGB1 mRNA expression was significantly increased at 10 weeks of age, which was followed by slow reduction in expression over time to reach levels similar to that at 8 weeks of age.

The NOD mice displayed much homogeneity in the expression of TLR4 mRNA during the development of diabetes. At 6 weeks of age, there was a distinct increase in TLR4 mRNA expression compared with that at 4 weeks of age. This increase was transient 


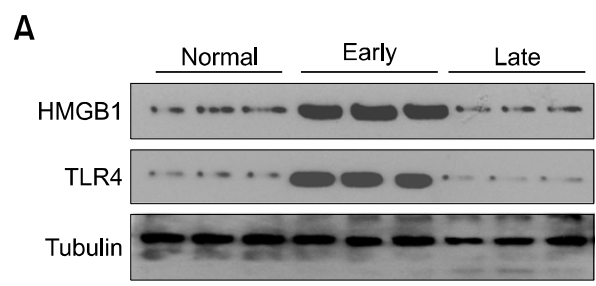

B

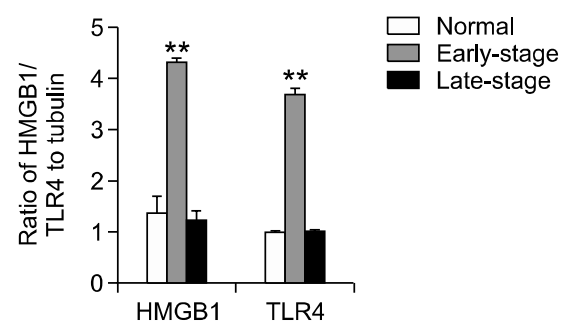

Figure 5. Western blotting analysis of HMGB1 and TLR-4 protein expression. Pancreatic expression of HMGB1 and TLR4 was low in non-diabetic NOD mice (4-6 weeks of age). In the early stage of diabetes (10-14 weeks of age), the expression of HMGB1 and TLR4 protein was significantly upregulated. In contrast, the expression of HMGB1 and TLR4 was decreased in mice with established diabetes (28-32 weeks of age). The data are from three independent experiments. ${ }^{* *} P<0.01$ versus control and late-stage normal mice.

and followed by a significant increase in TLR4 mRNA expression between 8 and 10 weeks of age, followed by a significant reduction in the values in later weeks.

\section{Discussion}

HMGB1 originated more than 500 million yr ago before the split between the animal and plant kingdoms (Han et al., 2008). Therefore, it is one of the most evolutionarily conserved proteins in the eukaryotic kingdom, shareing 99\% amino acid identity between rodents and humans (Han et al., 2008). HMGB1 can be either passively released by injured or necrotic cells or actively secreted by macrophages (Lin et al., 2011a). The release of HMGB1 into the extracellular environment, where it can function as an endogenous danger signal or 'alarmin' to promote inflammation, is implicated in several diseases such as sepsis and acute lung injury (Wang et al., 2010).

HMGB1 exerts pleiotropic effects by binding to several members of the toll-like family, including TLR2, TLR4, TLR9 and RAGE, and thus promotes inflammatory processes (Klune et al., 2008; Gdynia et al., 2010). TLRs are type 1 transmembrane receptors and recognize distinct pathogen-associated molecular patterns (PAMPs) through their leucine-

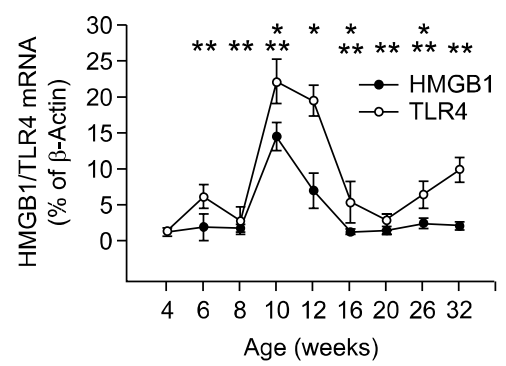

Figure 6. Pancreatic mRNA expression of HMGB1 and TLR-4 mRNA. Pancreatic tissue was obtained from NOD mice (8 mice per time) every 3-5 weeks from 4 to 32 weeks of age and subjected to qRT-PCR. Values are means \pm standard error of the mean. The data are from three independent experiments. ${ }^{*} P<0.05$ (HMGB1) for 8 versus 10 weeks of age, 10 versus 12 weeks of age, 12 versus 16 weeks of age and 20 versus 26 weeks of age (Wilcoxon's signed rank test). ${ }^{* *} P<0.05$ (TLR4) for 4 versus 6 weeks of age, 6 versus 8 weeks of age, 8 versus 10 weeks of age, 12 versus 16 weeks of age, 16 versus 20 weeks of age and 26 versus 32 weeks of age (Wilcoxon's signed rank test).

rich repeats in the extracytoplasmic domain (Miranda-Hernandez et al., 2011). TLRs can also recognize endogenous material during cellular injury (Johnson et al., 2003). The TLR family plays an essential role in innate and adaptive immune recognition in mammals. For example, TLR4 recognizes the Gram-negative product lipopolysaccharide and participates in the recognition of several endogenous ligands, including heat shock proteins, whereas TLR2 recognizes various fungal, Gram- positive and mycobacterial components (Yu et al., 2006). TLR9 recognizes bacterial CpG DNA sequences, and HMGB1 is involved in TLR9 activation, resulting in the maturation of immune cells and cytokine secretion (Klune et al., 2008; Miranda-Hernandez et al., 2011). Moreover, HMGB1-dependent activation of RAGE leads to activation of the NF- $\kappa B$ pathway, as well as signal transduction through ERK and p38, to promote cytokine production and cell survival (Lotze and Tracey, 2005).

T1DM is an autoimmune disease in which disordered innate immune response signaling is associated with disease progression. Current evidence suggests that abnormalities within the innate immune system play key roles in the pathogenesis of T1DM. In some people with T1DM, viral infection can lead to NK-dominant insulitis, $\beta$ cell dysfunction and eventually to overt hyperglycemia and diabetes (Grieco et al., 2011). The expression of TLR2 and TLR4, along with their downstream targets, was increased in monocytes obtained from patients with T1DM (Pino et al., 2010). Using a streptozotocin- induced diabetic C57BL/6 mouse model, Devaraj et al. found that genetic deficiency of TLR4 significantly reduced MyD88 and IRAK-1 protein phosphorylation and NF- $\mathrm{KB}$ activity, and attenuated the proin- 
flammatory state of T1DM (Devaraj et al., 2011).

The purpose of this study was to examine whether HMGB1 participates in the pathogenesis of T1DM and to identify the receptor involved in HMGB1-mediated activation of inflammatory signaling in NOD mice. First, we observed that the rate of HMGB1 expression in the cytoplasm of islets was much greater in diabetic mice compared with non-diabetic mice, which may be associated with islet destruction or insulitis. We proceeded to the expression of HMGB1 receptors (TLR2, TLR4, TLR9 and RAGE) on different pancreatic islet cells from non-diabetic NOD mice. We found that the majority of cells positively stained for TLR4 were $\beta$ cells, and these comprised the majority of cells within the islets. The expression of both HMGB1 and TLR4 was upregulated in the early stage of diabetes (8-10 weeks of age), which may hasten the development of diabetes. However, the expression of both HMGB1 and TLR4 was significantly decreased in the late stage of diabetes (16-32 weeks of age) compared with the early stage of diabetes (8-10 weeks of age), which may result from the destruction of the majority of $\beta$ cells within the islets and the reduction in the number of corresponding receptors. Our current findings indicate that TLR4 on $\beta$ cells could sense changes in HMGB1 released from necrotic islet cells, which may lead to a proinflammatory state around the islets and contribute to the initiation of autoimmune diabetes.

To our knowledge, this report is the first to describe a relationship between HMGB1 and its receptors on the isolated islets of NOD mice, an excellent model for T1DM in humans. We determined the changes that occur in pancreatic HMGB1 and TLR4 protein and mRNA expression throughout the course of diabetes in NOD mice. Our observations suggest that TLR4 is the main receptor on $\beta$ cells and that HMGB1 is passively released from damaged islet cells acts via TLR4 to selectively damage $\beta$ cells rather than $\alpha$ cells in the early stages of diabetes. Consequently, TLR4 may serve as an early marker for $\beta$ cell destruction, although studies of patients with T1DM are necessary to confirm this hypothesis. In future studies, we will examine TLR4 expression and signaling in type 2 diabetes, which is also a proinflammatory condition.

\section{Methods}

\section{Mice}

All animal experiments were performed in accordance with the guide for the Care and Use of Laboratory Animals approved by the Institutional Ethics Board of the Zhongshan Hospital of Fudan University. Healthy, 4-week-old (15-17 g) female NOD/JSlac mice were purchased from the Shanghai Laboratory Animal Center, Chinese Academy of Sciences. All the animals were housed in the specific pathogen-free animal facility of Zhongshan Hospital, Fudan University, Shanghai. Each mouse was weighed and the peripheral blood glucose level was monitored (A010120; Bayer, Germany) weekly before the animal was killed. Diabetes was diagnosed when two consecutive blood glucose measurements indicated values greater than 13.0 $\mathrm{mmol} / \mathrm{L}$ (Yaacob et al., 2009).

\section{Histological and morphological analyses}

Mice pancreases were excised and fixed overnight in $4 \%$ paraformaldehyde at $4^{\circ} \mathrm{C}$. Fixed tissues were processed for paraffin embedding and serial $5 \mu \mathrm{m}$ thick sections were prepared and stained with hematoxylin and eosin (Beyotime Institute of Biology, Suzhou, China) to assess pancreatic islets histology and morphology in the experimental animals. Histology and morphology of islets were scored with a Carl-Zeiss microscopy Axiovert 200 (Carl-Zeiss, Jena, Germany).

\section{Immunofluorescence and confocal analysis}

The pancreases of NOD mice were embedded with OCT Compound (Sakura Finetechnical Co., Ltd., Tokyo, Japan). Consecutive cryostat sections, $10 \mu \mathrm{m}$, were cut and air-dried at room temperature. The sections were first incubated overnight with primary antibody at $4^{\circ} \mathrm{C}$. Guinea pigs anti-mouse-insulin antibody (Invitrogen, Carlsbad, $\mathrm{CA}$ ), goat anti-mouse-glucagon antibody (Santa Cruz Biotechnology, Santa Cruz, CA), rabbit anti-mouse-HMGB1 antibody (Abcam, Cambridge, UK), mouse monoclonal T2.5 to TLR2 (Abcam), rabbit anti-mouse-TLR4 antibody (Abcam), mouse monoclonal 26C593 to TLR9 (Abcam) and rabbit anti-mouse-RAGE antibody (Millipore, Billerica, $\mathrm{MA}$ ) were the primary antibodies. The sections were washed three times with PBS and incubated for $2 \mathrm{~h}$ at room temperature with Cy3 conjugated donkey anti-GP secondary antibody (Millipore), Alexa Fluor 555 donkey anti-goat secondary antibody (Invitrogen), Alexa Fluor 488 goat anti-rabbit secondary antibody (Invitrogen), Alexa Fluor 555 goat anti-rabbit secondary antibody (Invitrogen) and Alexa Fluor 488 donkey anti-mouse secondary antibody (Invitrogen). The nuclear regions were stained by DAPI (Sigma-Aldrich) counterstaining. Finally, the sections mounted by glycerol/PBS (1:1). Isotype-matched antibodies and PBS were used as controls for stained sections. The samples were visualized on a Leica TCS SP5 confocal microscope (Leica, Mannheim, Germany).

\section{Separation and culture of islet cells}

The pancreas was distended via bile duct puncture and retrograde injection of $1.5-2 \mathrm{ml}$ of ice-cold collagenase type $\mathrm{V}$ (Sigma-Aldrich, St. Louis, MO). The gland was removed and placed into the D-Hank's balanced salt solution containing collagenase type $\mathrm{V}$. The pancreas was digested at $37^{\circ} \mathrm{C}$ under continuous shaking. The digest was washed three times with RPMI 1640 (Gibco, Incorporated, Invitrogen, Carlsbad, CA) containing $5 \%$ BSA and followed by Ficoll 
purification as described (Sutton et al., 1986). The separated islets were further purified by handpicking using capillary pipettes (BD, Franklin Lakes, $\mathrm{NJ}$ ) and were identified by selectively staining crimson red with Dithizone (DTZ) for the purity of the islets. Then, the dispersed islet cells were cultured in the standard medium, RPMI 1640 (Gibco) supplemented with 10\% fetal bovine serum (FBS) (Gibco), $20 \mathrm{mmol} / \mathrm{L}$ hydroxyethyl piperazine ethanesulfonic acid (HEPES) (Sigma-Aldrich), and $10 \mathrm{mmol} / \mathrm{L}$ niacinamide (Sigma-Aldrich) at $37^{\circ} \mathrm{C}$ in $95 \% \mathrm{O}_{2} / 5 \% \mathrm{CO}_{2}$ and saturated humidity.

\section{NHS-fluorescein labeling of HMGB1 and cellular immunofluorescence}

HMGB1 (eBioscience, San Diego, CA) protein was labeled with NHS-fluorescein (Pierce Biotechnology, Rockford, II) according to the manufacturer's instructions. Islet cells were isolated from 4-week-old non-diabetic NOD mice and plated in six-well plates (Corning Inc., Corning, NY). Cells were used at $70 \%$ confluence. Cells were preincubated with anti-TLR2, -TLR4, -TLR9 or -RAGE antibodies $(25 \mu \mathrm{g} / \mathrm{mL})$ for $30 \mathrm{~min}$ at $4^{\circ} \mathrm{C}$ in Opti-MEM I medium (Invitrogen). Cells were then treated with $5 \mu \mathrm{g} / \mathrm{mL}$ NHS-fluorescein-labeled HMGB1 and incubated for an additional $6 \mathrm{~h}$ at $4^{\circ} \mathrm{C}$ (Yu et al., 2006). Cells were then washed three times with PBS (pH 7.2) and fixed for $20 \mathrm{~min}$ at room temperature in $2 \%$ paraformaldehyde-PBS. After fixing, the fluorescence was recorded with a Leica TCS SP2 upright confocal microscope (Leica, Mannheim, Germany).

\section{Western blotting analysis}

Fifty micrograms of protein isolated from the pancreas of NOD mice was electrophoresed in $10 \%$ of sodium dodecyl sulfate-polyacrylamide gel electrophoresis gels and transferred onto polyvinylidene difluoride membranes (Millipore). After blocking with $5 \%$ non-fat milk, the membranes were incubated with primary antibodies recognizing HMGB1 (Abcam) and TLR4 (Abcam) overnight at $4^{\circ} \mathrm{C}$. Strips were then incubated with horseradish peroxidase (HRP)-conjugated goat anti-mouse and goat anti-rabbit secondary antibodies (Jackson ImmunoResearch Laboratories, West Grove, PA) for $1 \mathrm{~h}$ at room temperature. Proteins were visualized using enhanced chemiluminescence (ECL) western blotting detection reagents (Pierce) and quantified using a Bio-Rad Fluor-S Multi-Imager (Typhoon 9400, Amersham, Sweden).

\section{qRT-PCR analysis}

Total RNA ( $1 \mu \mathrm{g}$ from whole pancreas) was extracted and purified using TRIzol (Invitrogen) according to the manufacturer's instructions. Equal amounts of RNA were then reverse-transcribed into cDNA using a PrimeScript ${ }^{\circledR}$ RT reagent Kit (Takara Biotec, Kyoto, Japan). Quantitative realtime PCR was performed using the Mastercycler ${ }^{R}$ ep realplex real-time PCR system (Eppendorf, Hamburg, Germany) with SYBR green PCR master mix (Takara Biotec). The primers used for PCR were as follows: HMGB1, forward: GAGAGATGTGGAACAACACTGC, reverse: AGCTCTGTAGGCAGCAATATCC, 104 bp; TLR-4, forward: TTTCACC-
TCTGCCTTCACTACA, reverse: AGATACACCAACGGCTCTGAAT, 139 bp; $\beta$-actin, forward: GTCCCTCACCCTCCCAAAAG, reverse: GCTGCCTCAACACCTCAACCC, 266 bp. Cycling conditions were as follows: initial activation at $95^{\circ} \mathrm{C}$ for $30 \mathrm{~s}$; following 40 cycles for $5 \mathrm{~s}$ at $95^{\circ} \mathrm{C}$ and for 30 $s$ at $60^{\circ} \mathrm{C}$. The levels of target mRNA on cells were analyzed using the $2^{-\Delta \Delta \mathrm{Ct}}$ method.

\section{Statistical analysis}

Statistically significant differences between groups were assessed using parametric tests or non-parametric tests as appropriate. Values are presented as means \pm standard error of the mean. Statistical analysis was performed using SPSS 17.0 software. Values of $P<0.05$ were considered statistically significant.

\section{Acknowledgements}

This research was supported by a grant from the National Nature Science Foundation (30371388) and by the research funding scheme for the excellent doctoral students in key disciplines at Fudan University.

\section{References}

Abdulahad DA, Westra J, Limburg PC, Kallenberg C, Bijl M. HMGB1 in systemic lupus Erythematosus: Its role in cutaneous lesions development. Autoimmun Rev 2010;9: 661-5

Anderson MS, Bluestone JA. The NOD mouse: A model of immune dysregulation. Annu Rev Immunol 2005;23:447-85

Devaraj S, Tobias P, Jialal I. Knockout of toll-like receptor-4 attenuates the pro-inflammatory state of diabetes. Cytokines 2011;55:441-5

Gdynia G, Keith M, Kopitz J, Bergmann M, Fassl A, Weber AN, George J, Kees T, Zentgraf HW, Wiestler OD, Schirmacher P, Roth W. Danger signaling protein HMGB1 induces a distinct form of cell death accompanied by formation of giant mitochondria. Cancer Res 2010;70: 8558-68

Giarratana N, Penna G, Amuchastegui S, Mariani R, Daniel $\mathrm{KC}$, Adorini L. A vitamin $\mathrm{D}$ analog down-regulates proinflammatory chemokine production by pancreatic islets inhibiting T cell recruitment and type 1 diabetes development. J Immunol 2004;173:2280-7

Grieco FA, Vendrame F, Spagnuolo I, Dotta F. Innate immunity and the pathogenesis of type 1 diabetes. Semin Immunopathol 2011;33:57-66

Han J, Zhong J, Wei W, Wang Y, Huang Y, Yang P, Purohit S, Dong Z, Wang MH, She JX, Gong F, Stern DM, Wang CY. Extracellular high-mobility group box 1 acts as an innate immune mediator to enhance autoimmune progression and diabetes onset in NOD mice. Diabetes 2008;57:2118-27

Johnson GB, Brunn GJ, Platt JL. Activation of mammalian Toll-like receptors by endogenous agonists. Crit Rev Immunol 2003;23:15-44 
Klune JR, Dhupar R, Cardinal J, Billiar TR, Tsung A. HMGB1: Endogenous danger signaling. Mol Med 2008;14:476-84

Li M, Song L, Qin X. Glycan changes: cancer metastasis and anti-cancer vaccines. J Biosci 2010;35:665-73

Lin Q, Fang J, Fang D, Li B, Zhou H, Su SB. Production of recombinant human HMGB1 and anti-HMGB1 rabbit serum. Int Immunopharmacol 2011a;11:646-51

Lin Q, Yang XP, Fang D, Ren X, Zhou H, Fang J, Liu X, Zhou $S$, Wen F, Yao X, Wang JM, Su SB. High-mobility group box-1 mediates toll-like receptor 4-dependent angiogenesis. Arterioscler Thromb Vasc Biol 2011b;31:1024-32

Lotze MT, Tracey KJ. High-mobility group box 1 protein (HMGB) Nuclear weapon in the immune arsenal. Nat Rev Immunol 2005;5:331-42

Medzhitov R. Recognition of microorganisms and activation of the immune response. Nature 2007;449:819-26

Meyers AJ, Shah RR, Gottlieb PA, Zipris D. Altered toll-like receptor signaling pathways in human type 1 diabetes. $\mathrm{J} \mathrm{Mol}$ Med (Berl) 2010;88:1221-31

Miranda-Hernandez S, Gerlach N, Fletcher JM, Biros E, Mack M, Korner H, Baxter AG. Role for MyD88, TLR2 and TLR9 but Not TLR1, TLR4 or TLR6 in Experimental Autoimmune Encephalomyelitis. J Immunol 2011;187: 791-804

Nogueira-Machado JA, Volpe CM, Veloso CA, Chaves MM. HMGB1, TLR and RAGE: a functional tripod that leads to diabetic inflammation. Expert Opin Ther Targets 2011;15: 1023-35

Pino SC, Kruger AJ, Bortell R. The role of innate immune pathways in type 1 diabetes pathogenesis. Curr Opin Endocrinol Diabetes Obes 2010;17:126-30
Song JS, Kang CM, Park CK, Yoon HK, Lee SY, Ahn JH, Moon HS. Inhibitory effect of receptor for advanced glycation end products (RAGE) on the TGF-beta-induced alveolar epithelial to mesenchymal transition. Exp Mol Med 2011;43: 517-24

Sutton R, Peters M, Mcshane P, Gray D, Morris PJ. Isolation of rat pancreatic-islets by ductal injection of collagenase. Transplantation 1986;42:689-91

Van Beijnum JR, Buurman WA, Griffioen AW. Convergence and amplification of toll-like receptor (TLR) and receptor for advanced glycation end products (RAGE) signaling pathways via high mobility group B1 (HMGB1). Angiogenesis 2008;11:91-9

Wang F, Lu Z, Hawkes M, Yang H, Kain KC, Liles WC. Fas (CD95) induces rapid, TLR4/IRAK4-dependent release of pro-inflammatory HMGB1 from macrophages. J Inflamm (Lond) 2010;7:30

Wittemann B, Neuer G, Michels H, Truckenbrodt H, Bautz FA. Autoantibodies to nonhistone chromosomal-proteins hmg-1 and hmg-2 in sera of patients with juvenile rheumatoidarthritis. Arthritis Rheum 1990;33:1378-83

Yaacob NS, Kaderi MA, Norazmi MN. Differential transcriptional expression of PPAR alpha, PPAR gamma 1, and PPAR gamma 2 in the peritoneal macrophages and T-cell subsets of non-obese diabetic mice. J Clin Immunol 2009;29:595-602

Yu M, Wang H, Ding A, Golenbock DT, Latz E, Czura CJ, Fenton MJ, Tracey KJ, Yang H. HMGB1 signals through toll-like receptor (TLR) 4 and TLR2. Shock 2006;26:174-9

Zhang CC, Krieg S, Shapiro DJ. HMG-1 stimulates estrogen response element binding by estrogen receptor from stably transfected HeLa cells. Mol Endocrinol 1999;13:632-43 